\title{
Proyectos Comunitarios en el proceso de Transformación Social de la Universidad
}

\section{Community Projects in the process of Transformation Social of the University}

\author{
Denny Moraima Romero \\ moraimaromerop14@hotmail.com \\ Universidad Politécnica Territorial Alonso Gamero \\ Julio Juvenal Aldana Zavala \\ julioaldanazavala@gmail.com
}

Universidad Nacional Experimental Francisco de Miranda

https://orcid.org/0000-0002-7934-9103

\section{RESUMEN}

Los proyectos comunitarios consisten en pensar en un conjunto de actividades interrelacionadas que deben conducir al logro de los resultados esperados. De igual manera el proceso de transformación implica abordar una situación real y cambiarla en beneficio de un colectivo o de la sociedad, por ello es necesaria la participación activa de los involucrados. Además, es considerado en la actualidad una metodología dinámica en los procesos de formación de los estudiantes en las diferentes etapas de su desarrollo profesional y personal. En este sentido, este trabajo de investigación tiene como propósito transformar la participación organizada de los profesores responsables de proyectos comunitarios en la Universidad Politécnica Territorial "Alonso Gomero". Para ello se utilizó la modalidad de investigación acción participación, con una población de nueve (9) sujetos de investigación. La investigación se fundamenta bajo la concepción del paradigma socio crítico, que surge en respuesta a las tradiciones positivistas e interpretativas que han tenido poca influencia en la transformación social. Para realizar el diagnóstico y valoración de los resultados de las acciones aplicadas se utilizaron técnicas tales como la observación y las entrevistas abiertas, mediante instrumentos; registros y cuestionarios. Se elaboró y ejecutó un plan de acción, para 


\title{
CIENCIAMATRIA \\ Revista Interdisciplinaria de Humanidades, Educación, Ciencia y Tecnología \\ Año V. Vol. V. №9. Julio - Diciembre 2019 \\ Hecho el depósito de ley: pp201602FA4721 \\ ISSN-L: 2542-3029; ISSN: 2610-802X \\ Universidad Nacional Experimental Francisco de Miranda (UNEFM). Santa Ana de Coro. Venezuela \\ Denny Moraima Romero; Julio Juvenal Aldana Zavala
}

transformar la participación de los profesores responsables de proyectos comunitarios donde se obtuvo un cambio de actitud y reflexión en la participación activa de los proyectos.

Descriptores: Proyecto, proceso, transformación, seguimiento y control.

\begin{abstract}
Community projects consist in thinking about a set of interrelated activities that should lead to the achievement of the expected results. In the same way, the process of transformation implies addressing a real situation and changing it for the benefit of a group or society, for this reason the active participation of those involved is necessary. In addition, it is currently considered a dynamic methodology in the training processes of students in the different stages of their professional and personal development. In this sense, this research work has the purpose of transforming the organized participation of teachers responsible for community projects in the Polytechnic Territorial University "Alonso Gomero". To this end, the participatory action research modality was used, with a population of nine (9) research subjects. The research is based on the conception of the socio-critical paradigm, which arises in response to the positivist and interpretative traditions that have had little influence on social transformation. To make the diagnosis and evaluation of the results of the applied actions, techniques such as observation and open interviews were used, through instruments; records and questionnaires. An action plan was drawn up and implemented, to transform the participation of teachers responsible for community projects where a change of attitude and reflection in the active participation of the projects was obtained.
\end{abstract}

Descriptors: Project, process, transformation, monitoring and control.

\section{INTRODUCCIÓN}

La Constitución de la República Bolivariana de Venezuela (CRBV, 1999), sustenta una política de Estado destinada a orientar, consolidar, los procesos de transformación de los diferentes sectores involucrados en el desarrollo económico y social de la nación. Para ello es necesario generar alianzas estratégicas con los diferentes sectores de la 


\section{CIENCIAMATRIA \\ Revista Interdisciplinaria de Humanidades, Educación, Ciencia y Tecnología \\ Año V. Vol. V. №9. Julio - Diciembre 2019 \\ Hecho el depósito de ley: pp201602FA4721 \\ ISSN-L: 2542-3029; ISSN: 2610-802X \\ Universidad Nacional Experimental Francisco de Miranda (UNEFM). Santa Ana de Coro. Venezuela \\ Denny Moraima Romero; Julio Juvenal Aldana Zavala}

sociedad a través de una cultura de participación, una visión compartida y la formación de un ciudadano y una ciudadana que con su acción constituya las bases de una nueva sociedad multiétnica y pluricultural en el espíritu de la interculturalidad, valorando la participación ciudadana, tal como lo expresa la (CRBV, 1999) en su Artículo 62:

La participación del pueblo en la formación, ejecución y control de la gestión pública es el medio necesario para lograr el protagonismo que garantice su completo desarrollo, tanto individual como colectivo. Es obligación del estado y deber de la sociedad facilitar la generación de las condiciones más favorables para su práctica. (p. 3).

Desde esta perspectiva se considera la participación como el medio necesario para lograr el protagonismo que permita a la ciudadanía desarrollar una conciencia social comprometida con el desarrollo de las políticas sociales, garante de su adecuada implementación, mediante el ejercicio efectivo de su papel de contralora social.

La Constitución de la República Bolivariana de Venezuela (CRBV, 1999), fundamenta en la educación y el trabajo, el logro de sus fines, esto por cuanto la educación se concibe como un proceso integrado al desarrollo económico y social de la nación. En este enfoque, la universidad debe fortalecerse como espacio que propicie la participación protagónica junto a la familia y la comunidad, a través de una corresponsabilidad bien entendida en el proceso del desarrollo integral, tanto de la población atendida como de la institución que la circunda, entendiendo la comunidad como referencia del espacio social y cultural.

La formación universitaria en la actualidad está dada por la necesidad de vincular al estudiante con las problemáticas comunitarias. De allí que el Programa Nacional de Formación (PNF), es un método de educación universitaria creada e implementada en Venezuela, con la finalidad de proponer pautas, enfoques y modalidades en pensum de estudios de las carreras universitarias destinadas de la Misión Alma Mater. Tiene como 


\section{CIENCIAMATRIA \\ Revista Interdisciplinaria de Humanidades, Educación, Ciencia y Tecnología \\ Año V. Vol. V. №9. Julio - Diciembre 2019 \\ Hecho el depósito de ley: pp201602FA4721 \\ ISSN-L: 2542-3029; ISSN: 2610-802X \\ Universidad Nacional Experimental Francisco de Miranda (UNEFM). Santa Ana de Coro. Venezuela \\ Denny Moraima Romero; Julio Juvenal Aldana Zavala}

propósito principalmente la solución de problemas e interacción con el entorno y la colaboración comunitaria, así como el desarrollo integral del País.

De esta manera, la Universidad Politécnica Territorial “Alonso Gamero", viene aplicando políticas educativas que permitan el buen funcionamiento de la organización dentro y fuera de la institución; ejecutando proyectos comunitarios que están inmersos dentro de los planes de estudios de cada programa nacional de formación (PNF). Orientados básicamente por quienes forman parte de la Comunidad, en virtud de que son quienes conocen la situación real de la zona y sus habitantes, en este sentido, (Tovar, 2006) manifiesta que

El proyecto comunitario, consiste en un documento técnico basado en un proceso sistemático, para conocer la posibilidad de consolidar una iniciativa o propuesta de inversión de carácter social o productivo, que conlleve a mejorar el nivel de vida del entorno social y fortalezca la verdadera participación de las comunidades en los planes de desarrollo local, regional y nacional. (p. 15).

Esta definición explica cómo se puede transformar una situación real con la participación y colaboración de todos los actores involucrados en el proceso de investigación. De allí la gran importancia de los proyectos comunitarios en la organización y ejecución de actividades orientadas a satisfacer las necesidades más urgentes o apremiantes de una comunidad, en especial de las personas que habitan en el contexto social inherente al desarrollo de los proyectos comunitarios.

En tal sentido, el aprendizaje basado en desarrollos de proyectos socio comunitario de estudiantes articulados con las comunidades, representa un binomio que se debe aprovechar a los efectos de contar con conocimientos que beneficien a la población. Es por ello que la formulación participativa del proyecto comunitario, implica el esfuerzo que se concreta en los espacios comunitarios con la concurrencia de los vecinos, bajo una visión de compromiso colectivo, supone escuchar y recoger impresiones, sugerencias sobre los aspectos a contener en el proyecto. 


\section{CIENCIAMATRIA \\ Revista Interdisciplinaria de Humanidades, Educación, Ciencia y Tecnología \\ Año V. Vol. V. №9. Julio - Diciembre 2019 \\ Hecho el depósito de ley: pp201602FA4721 \\ ISSN-L: 2542-3029; ISSN: 2610-802X \\ Universidad Nacional Experimental Francisco de Miranda (UNEFM). Santa Ana de Coro. Venezuela \\ Denny Moraima Romero; Julio Juvenal Aldana Zavala}

En la actualidad, se tiene conciencia del gran sentido de identidad y vocación de los profesores universitarios, conociendo que la nación les demanda hoy grandes retos y tareas; en cuanto a la educación como proceso para la transformación de la sociedad, sin embargo, se ha podido observar que de alguna forma también se evidencia la resistencia al cambio manifestada por la incertidumbre que implica la nueva situación paradigmática en cuanto a la puesta en práctica de los proyectos comunitarios y su participación en el desarrollo de los mismos.

Desde lo planteado, se aborda la importancia de esta investigación y la aplicación de la propuesta para el desarrollo personal y profesional de los profesores asesores de proyecto comunitario, en cuanto a su cambio de actitud; desarrollando sentido de pertenencia, disposición a la formación, disposición al cambio, sensibilidad humana, trabajo en colectivo, receptividad ante la crítica y sugerencias, la responsabilidad en el cumplimiento de las políticas educativas, entre otros.

En la Universidad Politécnica Territorial Alonso Gamero es imprescindible motivar la participación organizada de los profesores asesores en la ejecución de los proyectos comunitarios, por cuanto se ha detectado algunas dificultades relacionadas con esta situación en observaciones realizadas a los diferentes programas de proyectos, todo esto se fundamenta en lo siguiente:

- Carecen de una planeación para la participación organizada: es decir; los profesores de proyecto en su mayoría no acompañan a los estudiantes a los territorios abordados.

- Se realizan visitas eventualmente a los territorios que no son suficientes para dar seguimiento y control: evidenciando así la baja motivación y el descontento por parte de los estudiantes; presentándose así debilidades que pueden ser arrastradas hasta el final de la ejecución del proyecto.

- Hasta el momento no se han realizado investigaciones sobre esta temática, lo que no ha permitido que atiendan debidamente las situaciones que afectan el cumplimiento de esta política educativa para el mayor beneficio de la sociedad. 


\section{CIENCIAMATRIA \\ Revista Interdisciplinaria de Humanidades, Educación, Ciencia y Tecnología \\ Año V. Vol. V. №9. Julio - Diciembre 2019 \\ Hecho el depósito de ley: pp201602FA4721 \\ ISSN-L: 2542-3029; ISSN: 2610-802X \\ Universidad Nacional Experimental Francisco de Miranda (UNEFM). Santa Ana de Coro. Venezuela \\ Denny Moraima Romero; Julio Juvenal Aldana Zavala}

- Existe resistencia al cambio de carreras a PNF, de tesis a proyectos y debilidad en el conocimiento en si de lo que es proyecto comunitario socio integrador.

Esta problemática hace necesario la aplicación de acciones para motivar la participación para que apliquen estas estrategias en su modo de actuación. Por todo lo antes expuesto, la investigación tuvo como propósito estimular la participación organizada de los profesores responsables de proyectos comunitarios y aplicar mecanismos de control, supervisión, en el proceso de los proyectos comunitarios como política educativa en la vinculación comunidad - universidad, para el buen funcionamiento en la parte organizacional de la institución, mediante de la aplicación de acciones que motivaran el interés de los responsables de promover el desarrollo de los proyectos comunitarios.

Estas acciones se planificaron desde la reflexión personal de cada uno de los responsables e involucrados, con la finalidad de generar el empoderamiento, el sentido de pertenencia y pertinencia de los profesores e integrantes de las comunidades en donde se desarrollan los proyectos comunitarios, vivenciándose así, desde la realidad la problemática a transformar para el bienestar de la población en general.

Para iniciar el diagnóstico en esta investigación como primera acción se estableció una comunicación escrita al Coordinador de la Sala de Proyecto, Profesor Manuel Capielo, con la finalidad de solicitar el permiso requerido para el estudio de situación problemática evidenciada mediante la observación empírica y que para obtener un diagnostico preliminar para luego entrar en el diagnóstico participativo; es necesario aplicarle a la población a estudiar una entrevista abierta con la finalidad de recoger información real sobre su participación organizada en la ejecución de los proyectos comunitarios, temática en la que está enmarcada el trabajo de investigación de la especialización en gerencia y liderazgo educativo.

Para realizar el análisis de la situación real se elaboró un instrumento tipo entrevista en donde se formularon cuatro preguntas en relación a su participación en los proyectos comunitarios: 


\section{CIENCIAMATRIA \\ Revista Interdisciplinaria de Humanidades, Educación, Ciencia y Tecnología \\ Año V. Vol. V. №9. Julio - Diciembre 2019 \\ Hecho el depósito de ley: pp201602FA4721 \\ ISSN-L: 2542-3029; ISSN: 2610-802X \\ Universidad Nacional Experimental Francisco de Miranda (UNEFM). Santa Ana de Coro. Venezuela \\ Denny Moraima Romero; Julio Juvenal Aldana Zavala}

¿Qué piensa sobre la aplicación de los proyectos comunitarios en el pensum de estudio?

¿Cómo planifica su participación en el desarrollo de los proyectos comunitarios?

¿Qué estrategia utiliza para lograr una comunicación efectiva entre los actores involucrados en los proyectos comunitarios y usted?

¿Cómo expresaría su vivencia en la ejecución de los proyectos comunitarios y su integración a la práctica?

Una vez formulado el instrumento se procedió a la realización de las entrevistas las cuales se planearon hacer en tres grupos por encuentro, por cuanto se requería la dedicación para poder registrar cada una de las respuestas en cada pregunta de forma precisa para luego realizar el análisis necesario para la elaboración del diagnóstico, en este primer encuentro se entrevistaron tres sujetos, en el segundo momento tres y en el tercer encuentro, los últimos tres sujetos del total de la muestra.

En el análisis de los instrumentos aplicados para recoger la información se pudo evidenciar que en la primera pregunta del cuestionario, siete de los entrevistados respondieron que esta herramienta pedagógica responde a aspectos políticos y que ellos cumplen en el desarrollo de los mismos por estar establecido en el pensum de estudios y ser responsables del programa de formación, los dos sujetos restantes respondieron que desconocen sobre el origen de esta iniciativa y que se dedican a cumplir con los lineamientos de la universidad.

En la segunda pregunta referida a la forma de abordaje y seguimiento de la aplicación de los proyectos comunitarios, una vez realizado el análisis se puede ver que sólo dos de los sujetos entrevistados manifestaron planear de forma eventual visitas a la comunidad donde se desarrolla el proyecto comunitario, cuatro de los sujetos expresaron que se conformaban con evidencias fotográficas para evaluar y controlar la ejecución de los proyectos comunitarios, y los tres restantes coincidieron en que los responsables son los estudiantes, siendo muy difícil realizar estas visitas de 


\section{CIENCIAMATRIA \\ Revista Interdisciplinaria de Humanidades, Educación, Ciencia y Tecnología \\ Año V. Vol. V. №9. Julio - Diciembre 2019 \\ Hecho el depósito de ley: pp201602FA4721 \\ ISSN-L: 2542-3029; ISSN: 2610-802X \\ Universidad Nacional Experimental Francisco de Miranda (UNEFM). Santa Ana de Coro. Venezuela \\ Denny Moraima Romero; Julio Juvenal Aldana Zavala}

participación en la comunidades por situaciones de tiempo, económicas y de transporte por el difícil acceso o por lo alejado de algunos territorios al recinto universitario.

En el análisis de la tercera pregunta sobre las estrategias utilizadas para una comunicación efectiva entre los responsables de ejecución de los proyectos comunitarios, coincidieron tres de los sujetos que se realiza un seguimiento mediante presentación de los avances, evidenciados en informe y fotografías, donde los estudiantes expresan lo que han hecho y lo que aspiran lograr, dos de los sujetos manifestaron que participan de forma eventual en algunas de las actividades relacionadas a la ejecución de los proyectos comunitarios, los cuatro restantes estuvieron de acuerdo en que la participación y comunicación de ellos con los estudiantes en el desarrollo de los proyectos estaba basada en la elaboración de un excelente informe y su presentación.

Analizando los resultados de la última y cuarta pregunta del cuestionario, se evidenció que sólo dos de los sujetos expresan alguna vivencia personal en las visitas realizadas durante la ejecución de los proyectos comunitarios, mientras que los siete restantes son incapaces de expresar vivencias, por cuanto nunca se han dado la oportunidad de apreciar y poner en práctica la valoración vivencial de la trasformación de una situación comunitaria, poniendo como excusas situaciones que demuestran el poco interés.

El análisis de los instrumentos permitió diagnosticar la problemática marcada por la baja participación de los profesores asesores responsables de proyectos comunitarios, situación que evidencia una irregularidad en su práctica profesional, por cuanto para el seguimiento, control y evaluación de la ejecución de proyectos, se requiere de un profesor comprometido, con lo que esto implica, así mismo el marco legal demanda como política educativa generar la participación protagónica no sólo de los estudiantes y la comunidad, sino de la institución universitaria a través de los profesores como mediadores del proceso. 


\section{CIENCIAMATRIA \\ Revista Interdisciplinaria de Humanidades, Educación, Ciencia y Tecnología \\ Año V. Vol. V. №9. Julio - Diciembre 2019 \\ Hecho el depósito de ley: pp201602FA4721 \\ ISSN-L: 2542-3029; ISSN: 2610-802X \\ Universidad Nacional Experimental Francisco de Miranda (UNEFM). Santa Ana de Coro. Venezuela \\ Denny Moraima Romero; Julio Juvenal Aldana Zavala}

\section{Propósito General de la Investigación}

Articular la participación organizada y protagónica de los profesores responsables de proyectos comunitarios en la Universidad Politécnica Territorial Alonso Gamero, sede Cumarebo del Estado Falcón.

\section{REFERENCIAL TEÓRICO}

\section{Proyectos comunitarios}

Los proyectos comunitarios se constituyen en una herramienta metódica para desarrollar trabajos que permitan contribuir en la transformación integral de las comunidades, los mismos pueden ser abordados por organizaciones comunitarias o por instituciones educativas, siendo en el caso de investigación, la universidad como centro generadora de los proyectos comunitarios. En este sentido, (la ley orgánica de los consejos comunales, 2009), define el proyecto comunitario del siguiente modo:

Es el conjunto de actividades concretas orientadas a lograr uno o varios objetivos, para dar respuesta a las necesidades, aspiraciones y potencialidades de las comunidades. Los proyectos deben contar con una programación de acciones determinadas en el tiempo, los recursos, los responsables y los resultados esperados. (p. 6).

Desde lo planteado, se evidencia la necesidad de contar con personas formadas para la correcta elaboración de los proyectos comunitarios, implicándose para tal fin un proceso sistemático que involucra al conjunto de personas que hacen vida en torno al contexto social en donde se diseña y pretende desarrollar el proyecto comunitario, para lo cual es importante aplicar metodologías que permitan masificar la participación protagónica de los actores sociales implicados, entendiéndose tal participación como un rol activo y transformador a lo largo del proceso de intervención comunitaria, situación que permite a los docentes universitarios, realizar investigaciones desde el contexto natural comunitario.

Para esto es indispensable que los docentes, estudiantes, en fin la universidad, se involucre activamente con la comunidad en pro de trabajar desde diversas ópticas 


\section{CIENCIAMATRIA \\ Revista Interdisciplinaria de Humanidades, Educación, Ciencia y Tecnología \\ Año V. Vol. V. №9. Julio - Diciembre 2019 \\ Hecho el depósito de ley: pp201602FA4721 \\ ISSN-L: 2542-3029; ISSN: 2610-802X \\ Universidad Nacional Experimental Francisco de Miranda (UNEFM). Santa Ana de Coro. Venezuela \\ Denny Moraima Romero; Julio Juvenal Aldana Zavala}

metódicas en el desarrollo de proyectos comunitarios, con la finalidad de abordar desde la complementariedad de saberes y conocimientos, la transformación sistemática de la comunidad participante en el proyecto comunitario. Por otro lado, (Portillo, 2003) define el proyecto comunitario como

Un documento escrito en el que se refleja un conjunto de actividades a realizar con el fin de resolver un problema en la comunidad. Los aspectos que como mínimo debe contener dicho documento son los siguientes: 1. Título y tipo de proyecto; 2. Justificación; 3. Objetivos; 4. Metas; 5. Actividades; 6. Localización y cobertura espacial; 7. Beneficiarios; 8. Ubicación y ejecución en el tiempo; 9. Recursos humanos; 10. Recursos materiales; 11. Seguimiento a la ejecución; 12. Evaluación final del proyecto; 13. Rendición de cuentas. (p. 108).

Por consiguiente, el proyecto comunitario es un instrumento en el cual se plantea la solución a un problema o la satisfacción de una necesidad sentida por la colectividad; es decir, es un plan de acción detallado que resuelve un problema, una necesidad colectiva, situacional, corresponsables y cogestionaria de la comunidad, de esta manera, a través de los proyectos comunitarios se puede avanzar en la construcción de una mejor sociedad; democrática, participativa y protagónica, que consolide valores de bien común, una sana y productiva convivencia.

\section{Ley de Servicio Comunitario del Estudiante de Educación Universitaria}

En la Gaceta Oficial № 38.272 del 14 de septiembre de 2005, en su Artículo 4, se define el servicio comunitario del estudiante de educación universitaria del siguiente modo:

A los efectos de esta Ley, se entiende por Servicio Comunitario, la actividad que deben desarrollar en las comunidades los estudiantes de educación universitaria que cursen estudios de formación profesional, aplicando los conocimientos científicos, técnicos, culturales, deportivos y humanísticos adquiridos durante su formación académica, en beneficio de la comunidad, para cooperar con su participación al cumplimiento de los fines del bienestar social, de acuerdo con lo establecido en la Constitución de la República Bolivariana de Venezuela y en esta Ley. (p. 1). 


\section{CIENCIAMATRIA \\ Revista Interdisciplinaria de Humanidades, Educación, Ciencia y Tecnología \\ Año V. Vol. V. №9. Julio - Diciembre 2019 \\ Hecho el depósito de ley: pp201602FA4721 \\ ISSN-L: 2542-3029; ISSN: 2610-802X \\ Universidad Nacional Experimental Francisco de Miranda (UNEFM). Santa Ana de Coro. Venezuela \\ Denny Moraima Romero; Julio Juvenal Aldana Zavala}

De allí que, la finalidad del servicio comunitario, es fomentar en el estudiante, la solidaridad y el compromiso con la comunidad como norma ética y ciudadana, hacer un acto de reciprocidad con la sociedad, enriquecer la actividad de educación superior, a través del aprendizaje servicio, con la aplicación de los conocimientos adquiridos durante la formación académica, artística, cultural y deportiva ,integrar las instituciones de educación superior con la comunidad, para contribuir al desarrollo de la sociedad venezolana.

El servicio comunitario es una contribución del sector universitario al "Capital Social del país", es decir, la suma de esfuerzos y voluntades para obtener mejores niveles de vida en la sociedad. En sus prácticas, los estudiantes aplicarán los conocimientos científicos, técnicos, culturales, deportivos y humanísticos adquiridos durante su formación académica.

\section{METODOLOGÍA DE LA INVESTIGACIÓN}

Se asumió el paradigma socio crítico, definido desde la óptica de Arnal (1992) como "el paradigma socio crítico adopta la idea de que la teoría crítica es una ciencia social, que no es puramente empírica ni solo interpretativa" (p. 28), por consiguiente desde este enfoque investigativo se generó una interacción entre los investigados y docentes universitarios asesores de proyectos comunitarios, en donde se procuró contribuir en articular una participación protagónica por parte de estos actores sociales en el desarrollo de los proyectos comunitarios, promoviéndose el intercambio de experiencias, conocimientos, los cuales permitieron trabajar progresivamente en la transcendencia de la problemática inicial planteada en la investigación.

La investigación se basó en el método de la investigación acción participativa que como sostiene (Silva, 2005), "está sujeta a las necesidades prioritaria de la situación objeto de estudio y a la constante autoevaluación que realizan los involucrados, como reflexión para encausar debidamente el proceso" (p. 156). De allí que, este proceso 


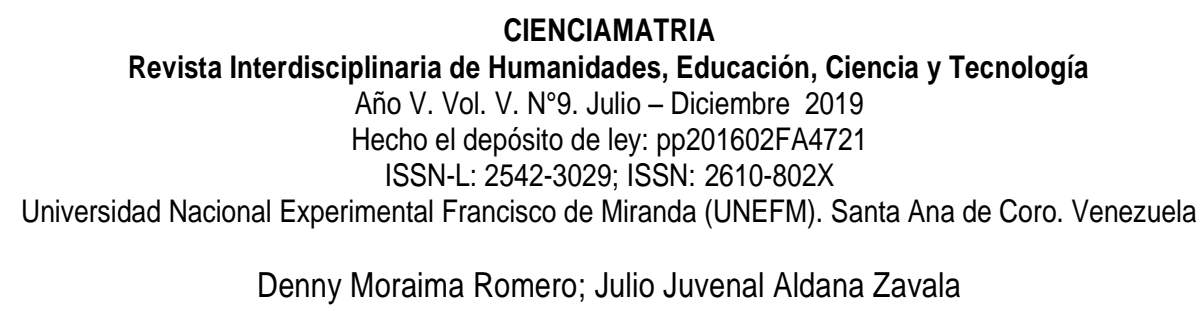

investigativo se sustentó desde el proceso reflexivo de sus actores sociales en virtud de las necesidades investigativas que se suscitaron durante el proceso.

En cuanto a los sujetos de investigación, se trabajó con nueve (9) profesores asesores de proyectos comunitarios de la Universidad Politécnica Territorial "Alonso Gamero" (UPTAG), sede Cumarebo del Estado Falcón, Venezuela, por cuanto es el escenario donde se desarrolla la participación de los actores sociales, fue necesario además la aplicación de técnicas cualitativas de investigación para la valoración del diagnóstico y el análisis de los resultados. En el desarrollo del proceso de investigación se utilizaron diferentes técnicas de investigación:

- Análisis y síntesis: se utilizó para el estudio, comprensión y reflexión de la fundamentación sobre la participación organizada de los profesores asesores de proyectos comunitarios.

- La Observación participante: se empleó durante todo el proceso de la investigación para evidenciar características y nivel de la participación de los profesores en el desarrollo de proyectos comunitarios.

- Análisis Documental: Se utilizó para analizar bibliografías y otros documentos.

- Entrevista: Se aplicó para diagnosticar la participación organizada para vivenciar el estado de ánimo y constatar la motivación de los profesores en la ejecución de los proyectos comunitarios.

\section{ANÁLISIS DE LOS RESULTADOS}

Los resultados de la investigación se basaron en función del desarrollo del siguiente plan de acción:

\section{Objetivo General del plan de acción}

Fomentar la participación protagónica y activa de los profesores responsables de proyectos comunitarios de la Universidad Politécnica Territorial Alonso Gamero, sede Cumarebo del Estado Falcón. 


\title{
Diseño del plan de acción
}

\author{
Cuadro 1 \\ Plan de Acción
}

\begin{tabular}{|c|c|c|c|c|c|}
\hline $\begin{array}{l}\text { OBJETIVOS } \\
\text { ESPECÍFICOS }\end{array}$ & ACCIONES & $\begin{array}{l}\text { RECURSOS, } \\
\text { TALENTOS }\end{array}$ & RESPONS & ES & $\begin{array}{l}\text { TIEMPO DE } \\
\text { EJECUCIÓN }\end{array}$ \\
\hline $\begin{array}{l}\text { Promover el } \\
\text { interés de los } \\
\text { profesores } \\
\text { asesores } \\
\text { responsables } \\
\text { de proyectos } \\
\text { comunitarios. }\end{array}$ & $\begin{array}{l}\text { Realizar charla } \\
\text { motivacional } \\
\text { reflexiva sobre la } \\
\text { participación }\end{array}$ & $\begin{array}{l}\text { Humano: } \\
\text { Profesional en el área } \\
\text { de la participación } \\
\text { Materiales: } \\
\text { Material digitalizado } \\
\text { Material impreso. }\end{array}$ & $\begin{array}{l}\text { Todos } \\
\text { responsable } \\
\text { proceso } \\
\text { investigación }\end{array}$ & $\begin{array}{l}\text { los } \\
\text { del } \\
\text { de }\end{array}$ & $\begin{array}{l}1 \text { Semana, para } \\
\text { la preparación, } \\
\text { desarrollo y } \\
\text { valoración. }\end{array}$ \\
\hline $\begin{array}{l}\text { Fomentar la } \\
\text { participación de } \\
\text { los profesores } \\
\text { asesores } \\
\text { responsables } \\
\text { de proyectos } \\
\text { comunitarios }\end{array}$ & $\begin{array}{l}\text { Desarrollar } \\
\text { conversatorio } \\
\text { sobre la } \\
\text { importancia de la } \\
\text { transformación } \\
\text { social. }\end{array}$ & $\begin{array}{l}\text { Humano: } \\
\text { Profesional en el área } \\
\text { de la transformación } \\
\text { social y su importancia. } \\
\text { Material: } \\
\text { Material impreso, video, } \\
\text { folletos. }\end{array}$ & $\begin{array}{l}\text { Todos } \\
\text { responsable } \\
\text { proceso } \\
\text { investigación }\end{array}$ & $\begin{array}{l}\text { los } \\
\text { del } \\
\text { de }\end{array}$ & $\begin{array}{l}1 \text { semana, para } \\
\text { la preparación, } \\
\text { desarrollo y } \\
\text { valoración. }\end{array}$ \\
\hline $\begin{array}{l}\text { Identificar la } \\
\text { sensibilidad de } \\
\text { los profesores } \\
\text { responsables } \\
\text { de proyectos } \\
\text { comunitarios }\end{array}$ & $\begin{array}{lr}\text { Presentar } & \text { video } \\
\text { sobre } & \text { una } \\
\text { experiencia } & \text { de } \\
\text { participación } & \text { en } \\
\text { la ejecución } & \text { de } \\
\text { proyecto } & \\
\text { comunitario } & \end{array}$ & $\begin{array}{l}\text { Humano: } \\
\text { Profesional en el área } \\
\text { de la Sociología } \\
\text { Materiales: } \\
\text { Material digitalizado } \\
\text { Material impreso. }\end{array}$ & $\begin{array}{l}\text { Todos } \\
\text { responsable } \\
\text { proceso } \\
\text { investigación }\end{array}$ & $\begin{array}{l}\text { los } \\
\text { del } \\
\text { de }\end{array}$ & $\begin{array}{l}1 \text { Semana, para } \\
\text { la preparación, } \\
\text { desarrollo y } \\
\text { valoración. }\end{array}$ \\
\hline $\begin{array}{l}\text { Impulsar la } \\
\text { convivencia } \\
\text { comunitaria de } \\
\text { los profesores } \\
\text { en la ejecución } \\
\text { de proyectos } \\
\text { comunitarios }\end{array}$ & $\begin{array}{ll}\text { Participar } & \text { en } \\
\text { acciones } & \text { de } \\
\text { ejecución } & \text { de } \\
\text { proyectos } & \\
\text { comunitarios } & \text { en } \\
\text { desarrollo } & \end{array}$ & $\begin{array}{l}\text { Humano: } \\
\text { Profesores, } \\
\text { estudiantes, comunidad } \\
\text { y consejos comunales. }\end{array}$ & $\begin{array}{l}\text { Todos } \\
\text { responsable } \\
\text { proceso } \\
\text { investigación }\end{array}$ & $\begin{array}{l}\text { los } \\
\text { del } \\
\text { de }\end{array}$ & $\begin{array}{l}3 \text { Semanas } \\
\text { para la } \\
\text { preparación, } \\
\text { desarrollo y } \\
\text { valoración. }\end{array}$ \\
\hline
\end{tabular}

Fuente: Investigadores, 2017

Todas las acciones ejecutadas para motivar la participación de los profesores asesores responsables de los proyectos comunitarios de la Universidad Politécnica "Alonso Gamero" (UPTAG), tuvieron una participación dinámica de grupo de profesores asesores, se pudo evidenciar el entusiasmo y las expectativas por involucrarse en cada una de las acciones que se planteaban a realizar en esta planeación. 


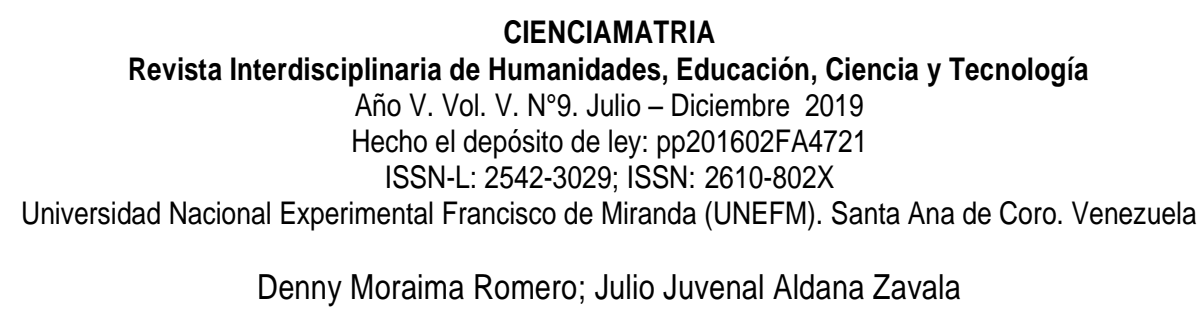

De igual manera se evidenció el final de cada acción la reflexión de cada participante observada en el modo de actuación y recogida en un instrumento aplicado al final de cada acción para valorar el impacto de la misma.

Se desarrolló una charla motivacional reflexiva para promover el interés de los profesores asesores responsables de proyectos comunitarios, se efectuó con la asistencia de los nueve profesores de proyecto de los diferentes programas, iniciando la actividad con el saludo de bienvenida y la introducción sobre el objetivo de la jornada del día, dando una lectura reflexiva sobre la práctica profesional.

Los profesores se mostraron a compartir en equipo el papel que juegan en la formación y transformación comunitaria, para concluir esta acción y valorar la efectividad de la misma se elaboraron conclusiones a partir de la experiencia al final se leyeron las conclusiones respectivas sistematizando y recogiendo los papeles de trabajo.

Para cumplir el objetivo para fomentar la participación de los profesores asesores responsables de proyectos comunitario se realizó un conversatorio sobre la importancia de la transformación social, se inició con el saludo de bienvenida a los presentes y presentación del especialista encargado de del conversatorio, en esta acción se enfatizó en la concepción de los proyectos comunitarios.

Tomando en cuenta la finalidad desde las políticas educativas en esta materia, para valorar el proceso reflexivo alcanzado por cada uno de los profesores participantes se les pidió elaborar de forma manuscrita un breve ensayo donde plasmaran desde su práctica profesional la concepción de un proyecto comunitario y el efecto en el territorio abordado, para finalizar cada profesor compartió con los compañeros su reflexión mediante la lectura de su ensayo.

La acción planteada identificar la sensibilidad de los profesores responsables de proyectos comunitarios se presentó video sobre una experiencia de participación en la ejecución de proyecto comunitario para detallar esta experiencia y su efecto en el colectivo participante sus motivaciones, para evaluar la acción se organizaron grupos para socializar la experiencia presentada en el video y elaborar conclusiones para 


\section{CIENCIAMATRIA \\ Revista Interdisciplinaria de Humanidades, Educación, Ciencia y Tecnología \\ Año V. Vol. V. №9. Julio - Diciembre 2019 \\ Hecho el depósito de ley: pp201602FA4721 \\ ISSN-L: 2542-3029; ISSN: 2610-802X \\ Universidad Nacional Experimental Francisco de Miranda (UNEFM). Santa Ana de Coro. Venezuela \\ Denny Moraima Romero; Julio Juvenal Aldana Zavala}

fortalecer la práctica personal en los proyectos comunitarios.

Para impulsar la convivencia comunitaria de los profesores en la ejecución de proyectos comunitarios se promovió entre los profesores una práctica dentro de la ejecución de los proyectos comunitarios que evidenciara el deber ser de su participación.

Para una evaluación general basada en la participación en las diferentes acciones ejecutadas en el plan de acción se pudo evidenciar lo siguiente:

- Motivación para elaborar desde ese momento una planificación dinámica y reflexiva para dar seguimiento y control a los proyectos comunitarios.

- Actitud positiva al cambio en su práctica profesional en relación a ejecución de los proyectos comunitarios.

- Valoración personal de la transformación social y personal que puede lograrse con los proyectos comunitarios.

- La iniciativa para investigar sobre los proyectos que actualmente se ejecutan como políticas educativas y su importancia para el beneficio de la sociedad actual y la formación del nuevo ciudadano y ciudadana que requiere el país.

En lo que respecta a las limitaciones encontradas se pueden mencionar las siguientes:

- El tiempo para poner en práctica las acciones, ya que al ver el interés de los actores involucrados en la dinámica de trabajo se evidenció que el tiempo planeado era poco.

- Dificultad en cuanto a la logística de traslado para las acciones en los territorios, pero que no impidieron que se cumplieran.

Es importante destacar que todas las dificultades encontradas fueron de carácter logístico y económico que se pudieron solventar en el proceso.

En la dinámica de transformación de la situación planteada en esta investigación se pudo vivenciar que cuando el ser humano se siente motivado desde la reflexión puede ser capaz de dar una mayor producción en su práctica profesional. 


\section{CIENCIAMATRIA \\ Revista Interdisciplinaria de Humanidades, Educación, Ciencia y Tecnología \\ Año V. Vol. V. №9. Julio - Diciembre 2019 \\ Hecho el depósito de ley: pp201602FA4721 \\ ISSN-L: 2542-3029; ISSN: 2610-802X \\ Universidad Nacional Experimental Francisco de Miranda (UNEFM). Santa Ana de Coro. Venezuela \\ Denny Moraima Romero; Julio Juvenal Aldana Zavala}

El carácter humanista de la educación debe dársele mayor énfasis, ya que los sentimientos y emociones afectan o favorecen en una determinada situación que pueda experimentar un profesor asesor en este caso.

\section{CONCLUSIONES}

El proceso seguimiento y control dentro del proceso de las diferentes áreas académicas y programadas de formación son esenciales para el desarrollo y ejecución de proyectos comunitarios. De allí que las acciones que conllevan siempre a una transformación deben valorarse constantemente para potenciar elementos que no puedan funcionar. En este sentido,

- El análisis realizado a la situación presentada en esta investigación permitió evidenciar que los profesores no se permitían la posibilidad de realizar acompañamientos a territorios abordados para realizar la transformación de la situación.

- La propuesta para transformar la situación real presentada se fundamenta en la participación efectiva de cada uno de los profesores responsables de proyectos para alcanzar la calidad de la ejecución de los mismos, mediante acciones dinámicas tales como charlas, mesas de trabajo, compartir de experiencias.

- La experiencia de las acciones aplicadas permitió el compartir de vivencias y realidades en los diferentes territorios abordados con cada uno de los PNF que se aplican, lo cual generó que es una necesidad la participación activa del profesor en los proyectos, que además de ser un requisito es una vivencia gratificante y satisfactoria como profesional y personal por estar acompañada de emociones y sentimientos dentro de los diferentes colectivos que hacen vida en los territorios.

\section{REFERENCIAS CONSULTADAS}

1. Arnal, J. (1992). Investigación educativa. Fundamentos y metodología. Barcelona (España): Labor. 


\author{
CIENCIAMATRIA \\ Revista Interdisciplinaria de Humanidades, Educación, Ciencia y Tecnología \\ Año V. Vol. V. №9. Julio - Diciembre 2019 \\ Hecho el depósito de ley: pp201602FA4721 \\ ISSN-L: 2542-3029; ISSN: 2610-802X \\ Universidad Nacional Experimental Francisco de Miranda (UNEFM). Santa Ana de Coro. Venezuela \\ Denny Moraima Romero; Julio Juvenal Aldana Zavala
}

2. Constitución de la República Bolivariana de Venezuela (1999). Capítulo IV. De los Derechos Políticos y del Referendo Popular. Sección Primera: De los Derechos Políticos. Artículo 62. Recuperado de http://www.cne.gov.ve/web/normativa electoral/constitucion/titulo3.php\#cap3

3. Ley Orgánica de Los Consejos Comunales, (2009). Capitulo I. disipaciones generales. Recuperado de http://www.minci.gob.ve/wpcontent/uploads/downloads/2012/11/LEY-CONSEJOS-COMUNALES-6-11-2012WEB.pdf

4. Ley de Servicio Comunitario del Estudiante de Educación Universitaria (2005). Capitulo II. Del servicio comunitario. Artículo 4. Recuperado de http://www.matematica.ciens.ucv.ve/files/Normativas/Ley deServicio Comunitario del Estudiante de Educacion Superior (2005).pdf

5. Silva, E. (2005). .Investigación Acción. Metodología transformadora.UNERMB, Santa Rita, Zulia, Venezuela.

6. Tovar, H. (2006). Modelo de Formulación de Proyectos Comunitarios. Guía para los Consejos Comunales. Universidad Rómulo Gallegos.

C2019 por los autores. Este artículo es de acceso abierto y distribuido según los términos y condiciones de la licencia Creative Commons Atribución-NoComercial-Compartirlgual 4.0 Internacional (CC BY-NC-SA 4.0) (https://creativecommons.org/licenses/by-nc-sa/4.0/). 